\title{
An Unusual Case of Small Bowel Obstruction
}

\author{
Darren J. Porter ${ }^{\mathrm{a}, \mathrm{b}}$, Charlotte Cosgrove ${ }^{\mathrm{a}}$, Emily Middleton ${ }^{\mathrm{a}}$
}

\begin{abstract}
Small bowel obstruction is a general surgical emergency usually caused by adhesions or herniae. Malignancy, inflammatory bowel disease and ingested foreign bodies (bezoars) are rarer causes. Bezoars can occur at any point in the gastrointestinal tract (GIT) depending on the type of bezoar and the point of impaction. Abdominal CT scan can assist in making an accurate and timely diagnosis of bezoar-induced small bowel obstruction. Bezoar-induced acute bowel obstruction requires either endoscopic or surgical extraction to avoid the complications of hemorrhage, perforation or fistula formation.
\end{abstract}

Keywords: Small bowel obstruction; Bezoar; Phytobezoar

\section{Introduction}

Acute small bowel obstruction is a common general surgical emergency usually resulting from adhesions or herniae. Bowel obstruction in a virgin abdomen is uncommon, with the main causes being malignancy, inflammatory bowel disease or ingested foreign bodies [1]. Ingested foreign bodies may be food or non-edible objects. Incidents of ingesting non-edible objects occur in children, elderly patients, psychiatric patients, prisoners and intoxicated individuals [2]. Bowel obstruction from edible items may occur in those with gastrointestinal motility disorders, Guillian-Barre syndrome, hypothyroidism and fast eaters [3]. Food items that have been reported to cause obstruction include dried fruit, apricot, coconut, raw banana and a whole lemon [3-5]. We report a case of acute small bowel obstruction requiring operative intervention in a 59-year-old

\footnotetext{
Manuscript accepted for publication October 16, 2015
}

aDepartment of General Surgery, Daisy Hill Hospital, Newry, County Down, Northern Ireland, UK

bCorresponding Author: Darren J. Porter, Department of General Surgery, Daisy Hill Hospital, Newry, County Down, Northern Ireland, UK.

Email: dporter@tcd.ie

doi: http://dx.doi.org/10.14740/jmc2343w lady caused by undigested vegetable.

\section{Case Report}

A 59-year-old lady was admitted via the emergency department with a $12 \mathrm{~h}$ history of colicky lower abdominal pain, bilious vomiting, constipation and abdominal distension. The patient had no history of previous surgery, Crohn's disease or diverticulosis. She took no regular medications, and had no family history of bowel pathology. Systematic review was unremarkable. Observations revealed a pulse rate of $95 \mathrm{bpm}$ and a temperature of $37.8^{\circ} \mathrm{C}$. On examination the patient had no evidence of recent weight loss, pallor or jaundice. On examination the abdomen was markedly distended, and there were no surgical scars and no abdominal wall or groin herniae. Palpation demonstrated a soft abdomen, with minimal tenderness in the right iliac fossa (RIF), percussion demonstrated a hyper-resonant abdomen and no bowel sounds were elicited on auscultation. PR examination was unremarkable. Admission bloods were notable only for an elevated white cell count (WCC) of $12.5 \times 10^{9} / \mathrm{L}$, hemoglobin, renal function, liver function, lactate and C-reactive protein were all normal. Plain film of the abdomen (PFA) showed dilated loops of small bowel with no air in the rectum (Fig. 1).

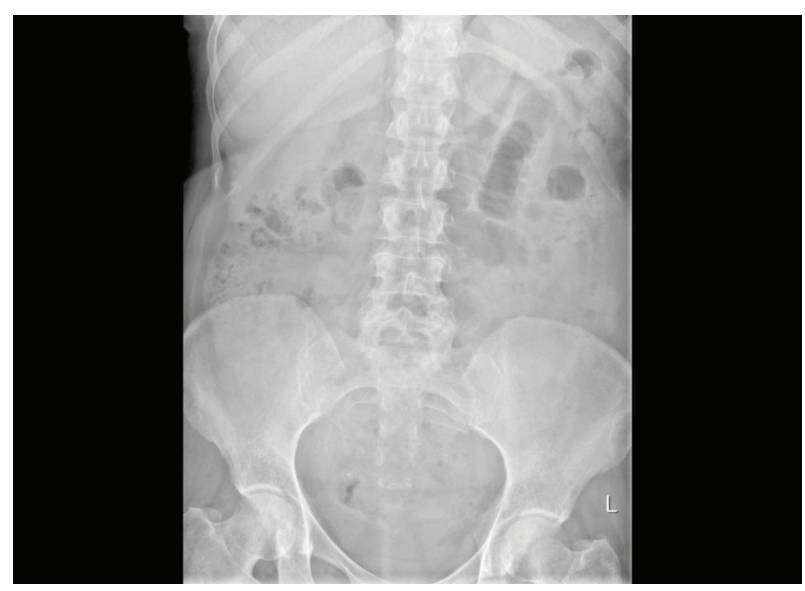

Figure 1. PFA demonstrating dilated small bowel loops and no air in the rectum. 


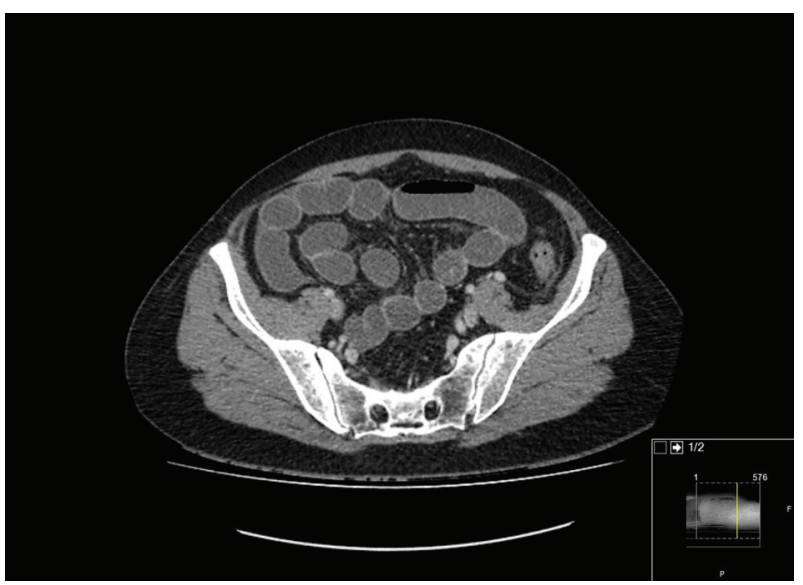

Figure 2. CT of abdomen and pelvis demonstrating marked small bowel dilatation with a transition point in the distal ileum.

PFA showed small bowel obstruction with uncertain etiology, a CT of abdomen and pelvis was requested and this demonstrated a transition point in the distal ileum with no obvious radiologically identifiable cause (Fig. 2).

A trial of conservative management was favored initially which included fasting the patient, nasogastric (NG) drainage, and intravenous fluids with electrolyte replacement; however, several hours later pain returned, and an emergency laparotomy was performed.

At laparotomy marked small bowel dilatation was evident to the point of obstruction in the distal ileum. Three foreign bodies were palpable within the distal ileum, and these were gently milked proximally. An enterotomy was performed and three pieces of undigested potatoes were delivered. The small bowel was then examined from the duodeno-jejunal flexure to the ileocecal valve. No strictures, adhesions, masses or other foreign bodies were found. No other abnormalities were demonstrated within the peritoneal cavity, and following a saline lavage the abdomen was closed. Post-operative recovery was uneventful, and the patient was discharged home on the fifth day. Prior to discharge the patient admitted to recently having had dentures fitted and acknowledged having difficulty adjusting to these and hence not chewing her food adequately.

\section{Discussion}

Small bowel obstruction is a common cause of general surgical admission and often requires surgical intervention. The common causes of small bowel obstruction are adhesions $(60 \%)$, external hernia (15\%), neoplasia $(6 \%)$ and inflammatory lesions $(5 \%)$. Rarely, in children, prison inmates and psychiatric patients, acute small bowel obstruction can be caused by ingested foreign bodies, including food bolus. Food bolus causing acute small bowel obstruction may occur in elderly patients with poor dentition and in those with dental prosthesis and inadequate mastication as seen in this case report [6].

A bezoar is a mass of undigested material which accumulates in the gastrointestinal tract (GIT), most often in the stom- ach. Bezoars can be classified according to the type of undigested material, and they include food bolus bezoar, phytobezoar (non-digestible particles from fruits and vegetables), lactobezoar (solidified milk), pharmacobezoar (pills and medications) and trichobezoar (hairballs) [2]. Although bezoars are well documented, their diagnosis and treatment remain a challenge [7].

Bezoars can occur at any point in the GIT and can be formed from a variety of substances, their presentation, investigation and management can vary greatly. The majority of bezoars pass through the GIT spontaneously, but if they get impacted in the GIT, they can cause obstruction, hemorrhage, perforation or fistula formation. Impaction typically occurs at sites of physiological narrowing in the GIT, for example, constrictions in the esophagus (arch of aorta, bronchus and at the lower esophageal sphincter), distal ileum and at the ileocecal valve or sites of pathological strictures [8]. The common presenting symptoms are chest pain, dysphagia and odynophagia if impaction occurs in the esophagus, symptoms of gastric outlet obstruction if impaction occurs in the stomach and colicky abdominal pain, bilious vomiting, abdominal distension and absolute constipation if the bezoar impacts in the small bowel. Investigations may include PFA which can diagnose acute intestinal obstruction with a high sensitivity (86\%), although it cannot detect bezoars which are radio-lucent [9]. Other useful investigations include barium swallow, CT scan and endoscopy. Barium studies may reveal mottling or a filling defect as the contrast infiltrates the bezoar. The abdominal CT scan is a most useful imaging modality, revealing the filling defect, the level where the bezoar is lodged and any complications such as obstruction or perforation [10]. Endoscopy can be both diagnostic and therapeutic. Small bezoars that might have been missed during imaging might be visualized during endoscopy. Certain organic bezoar can be managed non-invasively through dissolution with enzymatic therapy, but any bezoar causing acute bowel obstruction will require endoscopic or surgical extraction. Impacted enteral bezoars can be managed by open or laparoscopic approaches [3]. During surgery the bezoar can either be milked through the ileocecal valve into the cecum where it will subsequently pass in the stool; however, the majority of impacted bezoars need to be extracted via an enterotomy [8].

\section{Conclusion}

Small bowel obstruction due to bezoar is relatively uncommon. A high index of clinical suspicion must be present, as symptoms and signs are variable depending on the type of bezoar and the point of impaction. Abdominal CT scan can lead to a more accurate and timely diagnosis, and early surgery is advocated for bezoar-induced small bowel obstruction to reduce the rate of complications such as hemorrhage, perforation or fistula formation.

\section{Acknowledgement}

We would like to acknowledge the assistance of Mr. D. Gilpin, 
Consultant Surgeon, Daisy Hill Hospital for his invaluable contribution to this case report.

\section{Grant}

None.

\section{Conflict of Interest}

We have no conflicts of interest to declare.

\section{References}

1. Ooi S, Hong K. Small bowel obstruction caused by dried apple. Int J Surg Case Rep. 2015;10: 154-157.

2. Di Saverio S, Catena F, Coccolini F, Gazzotti F, Filicori F, Ansaloni L. Bizarre behaviour, bizarre intruder and bizarre bowel obstruction. BMJ Case Rep. 2010;2010.

3. Atila K, Guler S, Bora S, Gulay H. An unusual cause of small bowel perforation: apricot pit. Ulus Travma Acil Cerrahi Derg. 2011;17(3):286-288.

4. Slesak G, Mounlaphome K, Inthalad S, Phoutsavath O,
Mayxay M, Newton PN. Bowel obstruction from wild bananas: a neglected health problem in Laos. Trop Doct. 2011;41(2):85-90.

5. Seenu V, Iyer G, Rao CS. The Lemon Story: An Unusual Phytobezoar Causing Acute Small Bowel Obstruction. Oman Medical Journal. 2010:25(1):1-3.

6. Lohn JW, Austin RC, Winslet MC. Unusual causes of small-bowel obstruction. J R Soc Med. 2000;93(7):365368.

7. Michael KS. Bezoars: From Mystical Charms to Medical and Nutritional Management. Pract. Gastroenterol. 2002;13:37-50.

8. Eisen GM, Baron TH, Dominitz JA, Faigel DO, Goldstein JL, Johanson JF, Mallery JS, et al. Guideline for the management of ingested foreign bodies. Gastrointest Endosc. 2002;55(7):802-806.

9. Maglinte DD, Reyes BL, Harmon BH, Kelvin FM, Turner WW, Jr., Hage JE, Ng AC, et al. Reliability and role of plain film radiography and CT in the diagnosis of small-bowel obstruction. AJR Am J Roentgenol. 1996;167(6):1451-1455.

10. Altintoprak F, Degirmenci B, Dikicier E, Cakmak G, Kivilcim T, Akbulut G, Dilek ON, et al. CT findings of patients with small bowel obstruction due to bezoar: a descriptive study. ScientificWorldJournal. 2013;2013:298392. 